\title{
Anesthesia in a young adult with opsoclonus-myoclonus syndrome
}

\author{
Jeongwoo Lee, Deokkyu Kim, Byeongdo Jeon, Ji-Youn Oh, and Young Jin Han \\ Department of Anesthesiology and Pain Medicine, Chonbuk National University Medical School, Jeonju, Korea
}

Opsoclonus-myoclonus syndrome (OMS), also known as Kinsbourne syndrome or dancing-eye syndrome, is a very rare neurological disorder thought to be the result of autoimmune responses in the nervous system. It is characterized by opsoclonus (rapid, multidirectional, conjugate eye movements), myoclonus, ataxia, irritability, and sleep disturbance [1]. Here, we describe our experience of anesthetic management in an adult woman with OMS.

A 22 -year-old woman $(150 \mathrm{~cm}, 70 \mathrm{~kg})$ was referred for laparoscopic removal of a right ovarian cyst under general anesthesia. She had visited our neurology department after sudden onset of dizziness one month previously. Opsoclonus and myoclonus of the facial muscles were detected upon physical examination. Opsoclonus was confirmed by video-oculography, but a nerve conduction test revealed no electrophysiological abnormalities. Autoantibody titers were negative or in the normal range in serum and cerebrospinal fluid. No evidence of infection was found. $\left[{ }^{18} \mathrm{~F}\right]$-fluorodeoxyglucose (FDG) positron emission tomography-computed tomography imaging demonstrated low FDG uptake throughout both temporal and occipital lobes. Electroencephalography was normal without epileptiform discharges. Computed tomography imaging delineated a mature cystic mass of the right ovary. The patient was diagnosed with OMS. She was started on treatment with steroids and intravenous immunoglobulin, as well as symptom support therapy.

Before laparoscopic surgery, the patient was preoxygenated for $3 \mathrm{~min}$ in the operating room. Anesthesia was induced after placement of electrocardiogram (ECG) leads, a pulse oximeter, a blood pressure (BP) cuff, and a Bispectral index (BIS) monitor. The train-of-four (TOF) technique was applied to check the state of muscle relaxation during surgery. Initially, the patient's $\mathrm{BP}$ was 160/100 mmHg, her heart rate (HR) was 135 beats/min, her peripheral oxygen saturation $\left(\mathrm{SpO}_{2}\right)$ was $97 \%$, and her BIS was 98.

Propofol (Fresofol MCT 2\% inj., Fresenius Kabi, Graz, Austria) and remifentanil hydrochloride (GlaxoSmithKline, Verona, Italy) were administered at $4 \mu \mathrm{g} / \mathrm{ml}$ and $4 \mathrm{ng} / \mathrm{ml}$, respectively, using an effect-site concentration target-controlled infusion pump (Orchestra $^{\circledR}$, Fresenius Vial, Brezins, France). Both opsoclonus and myoclonus continued after the loss of consciousness until the target concentration of anesthetic drugs was reached. Then, $50 \mathrm{mg}$ of rocuronium was administered. When the TOF count reached 0 , opsoclonus and myoclonus disappeared, and the patient was successfully intubated.

The surgery lasted $60 \mathrm{~min}$. BP was held at 130-150/80-100 $\mathrm{mmHg}, \mathrm{HR}$ at $80-100$ beats/min, and BIS at 30-42 during the operation. The intraoperative TOF count remained at 0 . When the skin was closed, the TOF count was 2. A patient-controlled analgesia pump (ANAPA-plus with PCM, E-wha Fresenius Kabi, Gunpo, Korea) was applied for postoperative pain control with fentanyl and ketorolac. Five minutes after the beginning of skin closure, the TOF ratio reached $100 \%$ and myoclonus was detected on the patient's trunk. However, opsoclonus was not found. Once surgery was complete, infusion of propofol and remifentanil was stopped. Neostigmine $(1.5 \mathrm{mg})$ and glycopyrrolate $(0.4 \mathrm{mg})$ were injected to reverse muscle relaxantion.

Corresponding author: Young Jin Han, M.D., Ph.D., Department of Anesthesiology and Pain Medicine, Chonbuk National University Medical School, 20, Gunji-ro, Deokjin-gu, Jeonju 561-712, Korea. Tel: 82-63-250-1241, Fax: 82-63-250-1240, E-mail: yjhan@chonbuk.ac.kr

(c) This is an open-access article distributed under the terms of the Creative Commons Attribution Non-Commercial License (http:// creativecommons.org/licenses/by-nc/3.0/), which permits unrestricted non-commercial use, distribution, and reproduction in any medium, provided the original work is properly cited. 
Spontaneous ventilation was restored, and the patient was extubated and transferred to the post-anesthesia care unit (PACU). Upon arrival in the PACU, the patient was awake with stable vital signs.

BP, ECG, and BIS were also monitored in the PACU. Five minutes after arriving in the PACU, opsoclonus was detected in both eyes, which began to worsen. The patient was monitored in the PACU for an hour and her Aldrete score [2] was then found to be 10, at which point she was transferred to the ward. A pathological examination revealed a teratoma.

There have been few reports on the use of general anesthesia in adults with OMS.

Maranhao et al. [3] reported that sevoflurane and nitrous oxide anesthesia was successful for a child with OMS. However, in the current case, total intravenous anesthesia was chosen for anesthetic management. No adverse events occurred during anesthesia or the postoperative period. Thus, propofol, remifentanil, fentanyl and ketorolac were used safely for anesthetic management and pain control in a patient with OMS. It should be highlighted that ketamine and etomidate should be avoided in OMS patients, as they can exacerbate OMS symptoms [3].

OMS is a rare disease and its relationship with anesthesia has not been studied in detail. In cases of OMS, anesthesiologists should make a careful preoperative assessment and obtain documentation from the patient's neurologist outlining the course of the illness and explaining the disease process in order to make plans for safe anesthesia.

In conclusion, anesthetic management with propofol and remifentanil appears to be a safe option for OMS patients.

\section{References}

1. Kinsbourne M. Myoclonic encephalopathy of infants. J Neurol Neurosurg Psychiatry 1962; 25: 271-6.

2. Aldrete JA. The post-anesthesia recovery score revisited. J Clin Anesth 1995; 7: 89-91.

3. Maranhão MV, de Holanda AC, Tavares FL. Kinsbourne syndrome: case report. Rev Bras Anestesiol 2013; 63: 287-9. 